\title{
The Intracellular Growth of M. tuberculosis Is More Associated with High Glucose Levels Than with Impaired Responses of Monocytes from T2D Patients
}

\author{
Martha Torres, ${ }^{1}$ María Teresa Herrera, ${ }^{2}$ Guadalupe Fabián-San-Miguel, ${ }^{3}$ \\ and Yolanda Gonzalez $\mathbb{D}^{2}$ \\ ${ }^{1}$ Subdirección de Investigación Biomédica, National Institute of Respiratory Diseases, Mexico City, Mexico \\ ${ }^{2}$ Department for Microbiology Research, National Institute of Respiratory Diseases, Mexico City, Mexico \\ ${ }^{3}$ Clinic for Metabolic Syndrome, National Institute of Respiratory Diseases, Mexico City, Mexico \\ Correspondence should be addressed to Yolanda Gonzalez; yolag@hotmail.com
}

Received 24 December 2018; Revised 18 July 2019; Accepted 19 August 2019; Published 14 November 2019

Academic Editor: Francesca Santilli

Copyright (C) 2019 Martha Torres et al. This is an open access article distributed under the Creative Commons Attribution License, which permits unrestricted use, distribution, and reproduction in any medium, provided the original work is properly cited.

\begin{abstract}
Diabetes mellitus, a metabolic disease characterized by hyperglycemia and poor glucose control, is a risk factor for Mycobacterium tuberculosis (M. tuberculosis) infection and the development of active tuberculosis. To evaluate whether M. tuberculosis infection susceptibility is associated with an intrinsic factor in monocytes from type 2 diabetes (T2D) patients or it is associated with hyperglycemia per se, we analyzed TLR- 2 and TLR- 4 expression by flow cytometry and the cytokines IL-1 $\beta$, IL-6, IL-8, IL-10, and TNF- $\alpha$ by cytometric bead array assays, either stimulated with TLR-2 and TLR-4 ligands or infected with M. tuberculosis in the whole blood from T2D patients $(n=43)$ and healthy subjects $(n=26)$ or in CD14+ monocytes from healthy subjects cultured in high glucose (HG) $(30 \mathrm{mM})$. The intracellular growth of $M$. tuberculosis was evaluated by CFU counts at 0,1 , and 3 days in both monocytes from T2D patients and monocytes from healthy subjects cultured in HG. We did not find significant differences in TLR expression, cytokine production, or growth of M. tuberculosis in monocytes from T2D patients compared with those in monocytes from healthy subjects. Despite these results, in vitro assays of monocytes cultured with $30 \mathrm{mM}$ glucose led to significantly increased TLR-2 and TLR- 4 basal expression compared to those of monocytes cultured with $11 \mathrm{mM}$ glucose $(P<0.05)$. Conversely, the production of IL-6 by TLR-2 ligand stimulation, of IL-1 $\beta$, IL-6, and IL- 8 by TLR- 4 ligand stimulation, and of IL-8 by $M$. tuberculosis infection significantly decreased in monocytes cultured in $\mathrm{HG}(P<0.05)$. Additionally, the intracellular survival of $M$. tuberculosis increased in monocytes in HG after day 3 of culture $(P<0.05)$. In conclusion, HG decreased IL-8 production and the intracellular growth control of $M$. tuberculosis by monocytes, supporting the hypothesis that hyperglycemia plays an important role in the impaired immune responses to M. tuberculosis in patients with T2D.
\end{abstract}

\section{Introduction}

Diabetes mellitus (DM) represents a risk factor for Mycobacterium tuberculosis (M. tuberculosis) infection and the development of active tuberculosis (TB) [1]. Meta-analysis studies have shown that DM patients have an approximately 3 -fold higher risk of developing TB disease [2], and TB control treatment is difficult in these patients [3]. The risk of developing active TB among diabetic patients is particularly high in Hispanic people, perhaps because latent TB infection is more common in these populations [4]. The frequency of DM among patients with pulmonary TB is $29.63 \%$, and these patients have more severe clinical manifestations (i.e., a higher frequency of cavities on chest X-rays) and higher risks of recurrence and relapse than non-DM individuals with pulmonary TB [4], with poor glycemic control being a risk factor for TB [5].

$\mathrm{DM}$ is a metabolic disease characterized by hyperglycemia or high glucose $(\mathrm{HG})$ concentrations in blood resulting from defects in insulin secretion, insulin action, or both. Hyperglycemia induces a chronic inflammatory state through the secretion of proinflammatory cytokines in type 
TABLE 1: The clinical and demographic characteristics of the T2D patients and healthy subjects.

\begin{tabular}{|c|c|c|c|}
\hline & Healthy $(n=26)$ & $\mathrm{T} 2 \mathrm{D}(n=43)$ & ${ }^{*} P \leq 0.05$ \\
\hline Sex (male/female) & $9 / 17$ & $15 / 28$ & ns \\
\hline Age (years) & $48[33-65]$ & $53[30-68]$ & ns \\
\hline Weight (kg) & $65[51-95]$ & $66[51-114]$ & ns \\
\hline Height (m) & $1.58[1.45-1.80]$ & $1.56[1.44-1.74]$ & ns \\
\hline BMI $\left(\mathrm{kg} / \mathrm{m}^{2}\right)$ & $26[23-33]$ & $26[23-47]$ & ns \\
\hline Fasting glucose (mg/dl) & 99 [80-115] & 210 [106-460] & $*$ \\
\hline HbAlc (\%) & $5.5[5.0-5.9]$ & $8.9[6.1-15.6]$ & * \\
\hline HbAlc $>9(n=19)$ & - & $10.3[9.4-15.6]$ & \\
\hline Creatinine (mg/dl) & $0.77[0.59-1.10]$ & $0.71[0.52-1.84]$ & ns \\
\hline Cholesterol (mg/dl) & $186[136-313]$ & 200 [147-310] & ns \\
\hline Triglycerides & $132[70-469]$ & 215 [46-688] & * \\
\hline HDL (mg/dl) & 39 [26-67] & $43.2[29-70]$ & ns \\
\hline $\mathrm{LDL}(\mathrm{mg} / \mathrm{dl})$ & $124[76-208]$ & $125[11-228]$ & ns \\
\hline Status TST (+/-) & $47.8 \%(11 / 11)$ & $57.5 \%(23 / 17)$ & ns \\
\hline
\end{tabular}

T2D = type 2 diabetes mellitus, $\mathrm{BMI}=$ body mass index, $\mathrm{HbAlc}=$ glycated haemoglobin, $\mathrm{HDL}=$ high-density lipid, LDL = low-density lipid, TST = tuberculin skin test, $\mathrm{ns}=$ not significant. Values are median $[$ Min-Max]. $t$-test $=\mathrm{T} 2 \mathrm{D}$ vs. healthy. Two-sample proportion test for sex and TST.

2 diabetes (T2D) patients $[2,6]$. It is noteworthy that in spite of the activation state characteristic in these individuals, they still possess a higher susceptibility to developing infections by many pathogens, including $M$. tuberculosis [2] than non-T2D individuals, which makes it difficult to dissect and identify the participant mechanisms associated with the predisposition to infections seen in diabetic patients. Nevertheless, hyperglycemia has been suggested as an important factor in $M$. tuberculosis infection susceptibility [6].

In physiological conditions, the mechanism of inflammation through the activation of monocytes and macrophages results after pathogen recognition by protein receptors, such as the toll-like receptor (TLR) family. HG concentrations are able to upregulate TLR-2 and TLR- 4 and activate nuclear factor $-\kappa \mathrm{B}(\mathrm{NF}-\kappa \mathrm{B})$ to induce proinflammatory cytokine production in THP1 monocytes in the absence of pathogens [7]. TLRs play a critical role in both innate immune responses and the initiation of adaptive immunity to $M$. tuberculosis, specifically TLR-2 and TLR-4. TLR activation upregulates the transcription of proinflammatory cytokines, including IL-1 $\beta$, IL-6, and TNF- $\alpha$, which are essential for the recruitment of immune cells to the site of infection and the control of M. tuberculosis infection [8].

In monocytes from T2D patients, TLR-2 and TLR-4 expression is increased $[9,10]$, and these patients present chronic low-grade inflammation with secretion of TNF- $\alpha$ and IL-8. The proinflammatory cytokines are induced by different mechanisms, such as TLR-2/4 activation (TLR-4 activation is induced by elevated exogenous ligands, such as dietary fatty acids and enteric lipopolysaccharide, and endogenous ligands, such as free fatty acids, which are elevated in obese states), reactive oxygen species (ROS) generation, or CD33 downregulation [9, 11-13]. Those mechanisms are important for pathogen control; however, the ROS and proinflammatory cytokines induced by $\mathrm{HG}$ do not protect against infections. In contrast, T2D patients are more susceptible than non-T2D individuals to infections, including $M$. tuberculosis infection, suggesting an immune susceptibility induced by HG concentrations.

The immunologic mechanisms of susceptibility to $M$. tuberculosis in T2D patients are still not fully understood. To investigate whether susceptibility to tuberculosis is due to an intrinsic alteration of monocytes from T2D patients, we used a whole-blood assay to preserve the physiological environment. To address whether the enhanced susceptibility to tuberculosis is due to HG concentrations per se, we used an in vitro model of hyperglycemia (using $30 \mathrm{mM}$ glucose). In both assays, TLR-2 and TLR-4 expression, the cytokines released after TLR activation, and the growth control of $M$. tuberculosis were evaluated.

\section{Materials and Methods}

2.1. Study Population. Forty-three T2D patients were recruited from the Metabolic Syndrome Clinic of the National Institute of Respiratory Diseases in Mexico City according to the diagnosis criteria of the American Diabetes Association (Diagnosis and Classification of DM) [14]. Twenty-six healthy subjects (based on clinical laboratory tests) were invited to participate in this study. The Ethics Committee of the National Institute of Respiratory Diseases (INER) approved this study, and all participants provided written informed consent. Routine laboratory tests on blood as well as chest X-rays and Mantoux tuberculin skin tests (TSTs) were conducted (Table 1). Additionally, buffy coats from healthy adult donors were kindly provided by the blood banks at the INER.

2.2. High-Glucose Medium. Unless otherwise specified, all cells were cultured in RPMI 1640 medium according to formula specification with $11 \mathrm{mM}$ glucose (Cambrex, Walkersville, MD, USA) that was supplemented with $50 \mu \mathrm{g} / \mathrm{ml}$ gentamicin sulfate, $2 \mathrm{mM}$ L-glutamine, and $10 \%$ heatinactivated pooled human serum (Gemini Bioproducts, 
Sacramento, CA, USA) at $37^{\circ} \mathrm{C}$ in a $5 \% \mathrm{CO}_{2}$ atmosphere. Culture medium with $30 \mathrm{mM}(540 \mathrm{mg} / \mathrm{dl}) \mathrm{D}$-glucose (Sigma, St. Louis, MO, USA) is referred to in the text as HG.

2.3. Human Peripheral Monocyte Isolation. Sixty milliliters of heparinized human peripheral venous blood was obtained from the participants, and peripheral blood mononuclear cells were obtained from whole blood by centrifugation using Lymphoprep $^{\circledR}$ (Nycomed Pharma, Oslo, Norway). Monocytes were positively selected by using MACS ${ }^{\circledR}$ magnetic beads coupled to an anti-human CD14 antibody (Miltenyi Biotec, Auburn, CA, USA) according to the manufacturer's recommendations. Purity was assessed by conventional flow cytometry; preparations exhibited $>90 \%$ monocytes. Cell viability was assessed by trypan blue exclusion in all experiments and was $>95 \%$.

2.4. Mycobacterial Preparation. The M. tuberculosis strain H37Ra (25177, ATCC, Manassas, VA) was grown to a log phase in Middlebrook 7H9 broth (Difco Laboratories, Detroit, MI) supplemented with 1\% glycerol and 10\% albumin dextrose catalase enrichment (ADC, Becton Dickinson Co., MD) on an orbital shaking incubator at $37^{\circ} \mathrm{C}$. Mycobacteria were harvested and frozen at $-80^{\circ} \mathrm{C}$ until use ("stock bacteria"). For the infection assay, "stock bacteria" were thawed, and to remove any remaining large clumps, bacterial pellets were homogenized by passing them through a 26-gauge needle and centrifuged. The bacterial concentration was determined by serial dilution, and colony-forming units (CFU) were counted on Difco Middlebrook 7H10 agar plates (Becton Dickinson Co, MD) after disruption of mycobacterial clumps. M. tuberculosis was dyed with fluorescein diacetate (Fluka, Chemical, Switzerland) in $1 \mathrm{ml}$ of phosphatebuffered saline ( $\mathrm{pH} 9.1)$ for $30 \mathrm{~min}$ at $4^{\circ} \mathrm{C}$.

2.5. TLR Surface Expression by Flow Cytometry. The whole blood from T2D and healthy subjects or CD14+ monocytes from healthy subjects cultivated in vitro with $\mathrm{HG}$ were stained with phycoerythrin- (PE-) labeled anti-human TLR2, TLR-4, and CD14-FITC antibodies (eBioscience, San Diego, CA) or isotype controls. Cells were fixed with $1 \%$ paraformaldehyde, and TLR-2 and TLR-4 surface expression was analyzed in the monocyte region by flow cytometry. The results were analyzed as the percentage of positive cells and by mean fluorescence intensity (MFI) based on 10,000 events acquired in a FACSCanto II cytometer (BD). FlowJo software version 10.4.1 Mac OS X was used for analysis.

2.6. Cytokine Production in Response to TLR Ligands. One milliliter of whole blood from T2D and healthy subjects or $0.5 \times 10^{6} \mathrm{CD} 14+$ monocytes/well placed in 24-well plates were cultured in $\mathrm{HG}(30 \mathrm{mM})$ for $24 \mathrm{~h}$ and infected with $M$. tuberculosis (MOI of 10) or stimulated with $1 \mathrm{ng} / \mathrm{ml}$ TLR-2 ligand, synthetic lipoprotein palmitylated N-acyl-S-diacylglyceryl cysteine (Pam ${ }_{3}$ Cys; EMC Microcollections, Tuebingen, Germany), or $100 \mathrm{ng} / \mathrm{ml}$ TLR-4 ligand, Escherichia coli lipopolysaccharide (LPS; Sigma), and were further incubated for $4 \mathrm{~h}$ for T2D and healthy subjects or $24 \mathrm{~h}$ for monocytes in HG. Supernatants were collected, and interleukin-1 $\beta$ (IL- $1 \beta$ ), interleukin-6 (IL-6), interleukin-8 (IL-8), interleukin-10 (IL-
10), and tumor necrosis factor- $\alpha$ (TNF- $\alpha$ ) levels were measured by a cytometric bead array (BD Bioscience) following the manufacturer's recommendation. CellQuest software was used for sample acquisition, and data were formatted using BD Bioscience CBA software. The results were based on a standard concentration curve, and the cytokine concentration was expressed as $\mathrm{pg} / \mathrm{ml}$.

2.7. M. tuberculosis Growth Assay. CD14+ monocytes from T2D patients or healthy subjects were resuspended in RPMI 1640 medium, or monocytes from healthy subjects were resuspended in HG medium and cultured for $24 \mathrm{~h}$. Then, $10^{5}$ monocytes/well were plated in 96-well flat plates (Corning Inc., Corning, NY) in triplicate and infected with $M$. tuberculosis H37Ra (MOI of 10) for $1 \mathrm{~h}$. After removal of extracellular (nonphagocytosed) mycobacteria, RPMI or HG medium was added, and intracellular M. tuberculosis growth was determined. Briefly, after 0,1 , and 3 days of culture, cells were lysed with $0.1 \%$ sodium dodecyl sulfate (Sigma) for $10 \mathrm{~min}$ and then neutralized with $20 \%$ bovine serum albumin (Sigma). The lysates were serially diluted and plated in triplicate into Middlebrook 7H10 agar and incubated for 21 days at $37^{\circ} \mathrm{C}$ in $5 \%$ humidified $\mathrm{CO}_{2}$. The $\mathrm{CFU}$ were counted and reported as $\mathrm{CFU} / \mathrm{ml}$. In a set of control experiments, cell viability was assessed via a trypan blue exclusion assay ( $>90 \%)$ on days 0,1 , and 3 to estimate cell death as a result of treatment.

2.8. Statistical Analysis. Comparisons between T2D patients and healthy subjects or between RPMI and HG were made using the nonparametric Mann-Whitney rank sum test with GraphPad Prism 6.0h for Mac (GraphPad Software Inc.). The median with range is shown in the table, and the median is shown in all the figures. Statistical significance was set at $P<0.05$.

\section{Results}

3.1. Patients and Healthy Subjects. The clinical and demographic characteristics of the T2D patients and healthy subjects are summarized in Table 1. No significant differences in sex, age, or levels of creatinine or HDL or LDL cholesterol between T2D patients and healthy subjects were observed; however, both groups were overweight. The levels of glucose, HbAlc, and triglycerides were significantly higher among T2D patients than among healthy subjects $(P<0.05)$.

3.2. The Expression of TLR-2 and TLR-4 in Monocytes from T2D Patients Is Similar to That in Monocytes from Healthy Subjects. TLRs are essential for pathogen recognition. To analyze the expression of TLR-2 and TLR-4 cell surface receptors in monocytes from T2D patients, we evaluated the TLR-2 and TLR-4 expression in the monocyte region in the whole blood from T2D patients by flow cytometry. We do not find any differences in the T2D group vs. healthy subjects; because of this, we analyzed a subgroup with an HbAlc of $9 \%$ and above (poor glycemic control). As shown in Figures 1(a) and 1(b), TLR expression in T2D and healthy subjects was assessed on monocytes, and the cell surface expression of 


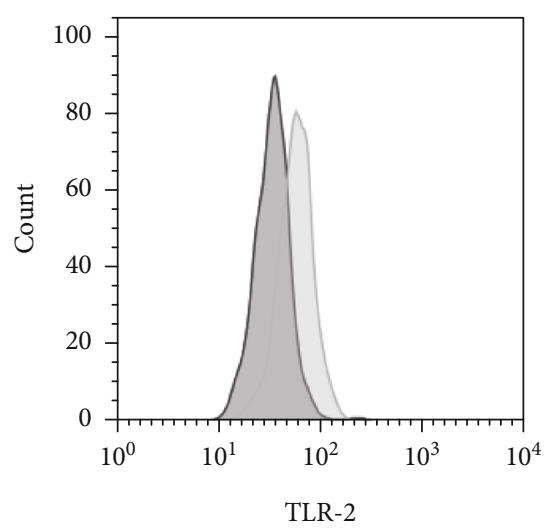

(a)

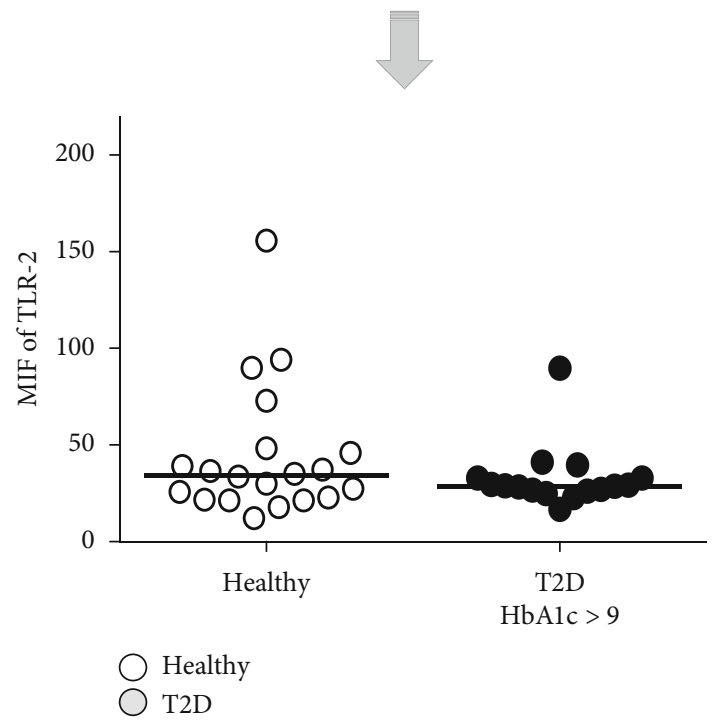

(c)

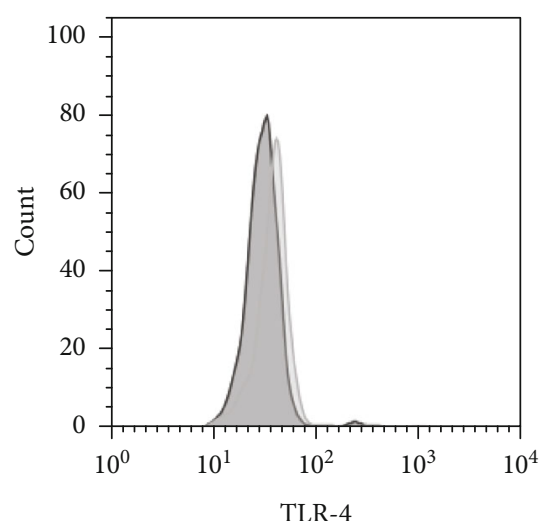

(b)

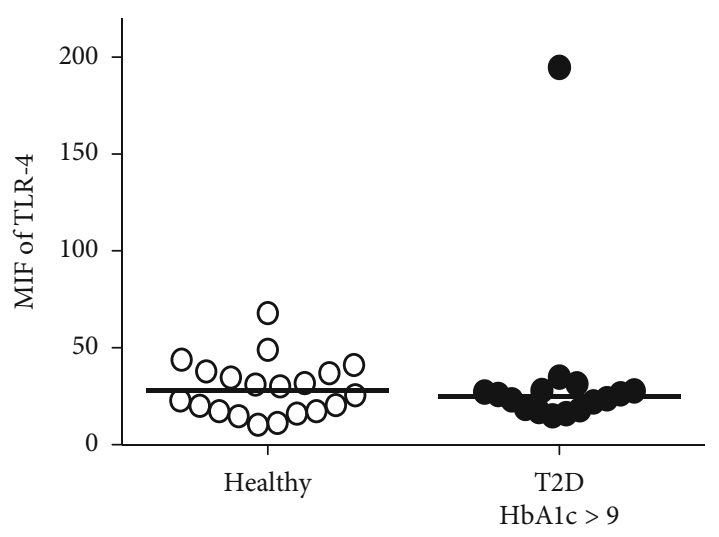

(d)

FIGURE 1: TLR cell surface expression in monocytes in T2D patients and healthy subjects. TLR surface expression was analyzed in whole blood samples by surface staining with AbM anti-human TLR-2PE or TLR-4PE and analyzed by flow cytometry. The monocyte region was analyzed, and TLR expression was reported as MFI. A representative experiment examining (a) TLR-2 and (b) TLR-4 in T2D and healthy subjects by cytometry is shown. Additionally, the surface expression of (c) TLR-2 and (d) TLR-4 in healthy subjects (open circle, $n=20$ ) and T2D patients with $\mathrm{HbAlc}>9$ (closed circle, $n=16$ ) is shown. The median is represented by a horizontal line.

TLR-2 (median, 28.7 for T2D and 34.3 for healthy) and TLR4 (median, 24.8 for T2D and 27.9 for healthy) was similar in monocytes from T2D and healthy subjects (Figures 1(c) and $1(d))$. The percentage of monocytes expressing TLR-2 and TLR-4 in healthy and T2D patients was similar in both groups (data not shown).

3.3. Cytokine Production in Response to TLR-2 and TLR-4 Activation or M. tuberculosis Infection Is Similar in T2D and in Healthy Subjects. To analyze TLR activation in monocytes from T2D patients and healthy subjects, further experiments were conducted to evaluate the IL- $1 \beta$, IL-6, IL-8, IL10 , and TNF- $\alpha$ levels induced by TLR ligands, Pam3Cys or LPS, or M. tuberculosis infection in monocytes from T2D patients with an $\mathrm{HbA} 1 \mathrm{c}$ of $9 \%$ and above (poor glycemic control) and healthy subjects. Cytokine production was determined after stimulation with Pam3Cys (median (pg/ml), IL$1 \beta-0$ and 32.9, IL-6-1058.9 and 1600.7, IL-8-1529.2 and 1740.6, and TNF- $\alpha-193.6$ and 402.1 for T2D and healthy, respectively), LPS (median (pg/ml), IL-1 $\beta-213.4$ and 246.0, IL-6-11762.8 and 21460.7, IL-8-2382.5 and 1030.6, IL$10-0$ and 7.2 , and TNF- $\alpha-1830.6$ and 1660.9 for T2D and healthy, respectively), and $M$. tuberculosis infection (median (pg/ml), IL-1 $\beta-23.3$ and 153.0, IL-6-827.1 and 2673.6, and IL-8-913.3 and 1832.8 for T2D and healthy, respectively). Lower IL-10 production was observed in T2D only after Pam3Cys stimulation (median (pg/ml), $0.0(0-25.3)$ in T2D and 6.5 (0-139.5) in healthy subjects) and after M. tuberculosis infection (median (pg/ml), 0.0 (0-6.1) in T2D and 0 (0-270) in healthy subjects $)(P<0.05$, Figures $2(\mathrm{a})-2(\mathrm{c}))$. We analyzed the correlation of cytokines with BMI; for T2D patients, no correlation was found $(P>0.05)$, and for healthy subjects, just TNF- $\alpha$ and BMI correlate weakly after LPS stimulation $(P>0.042, r=0.535)$ (Figure S1).

3.4. The M. tuberculosis Growth in Monocytes from T2D Patients Is Similar to That in Monocytes from Healthy Subjects. Since T2D patients with poor glycemic control show 


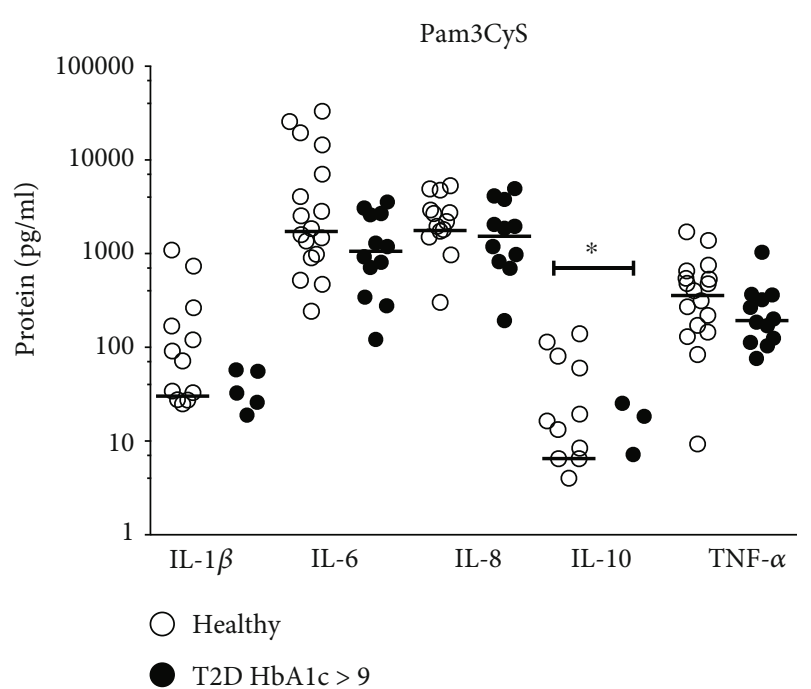

(a)

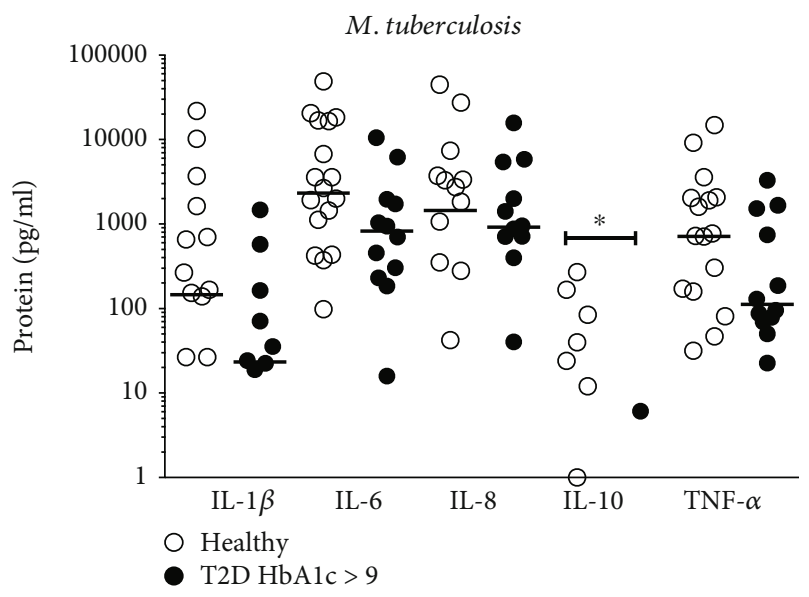

(c)

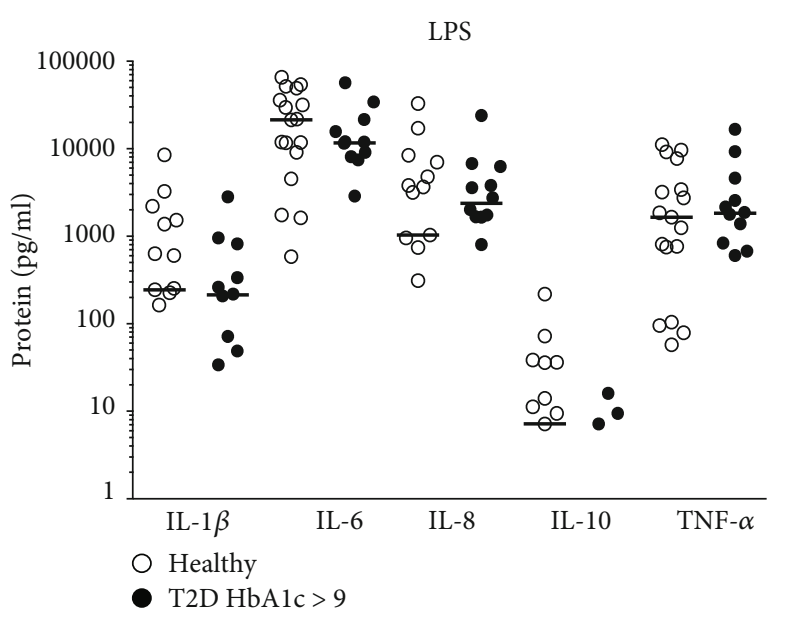

(b)

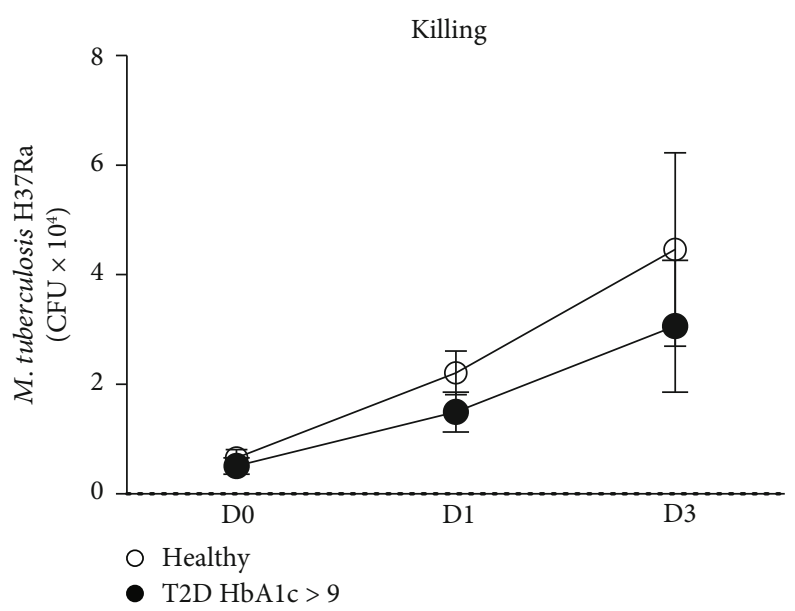

(d)

FIGURE 2: Cytokine production after TLR-specific stimulation or M. tuberculosis infection and intracellular growth control in monocytes from T2D patients and healthy subjects. Cytokine production elicited by activation through TLRs or $M$. tuberculosis infection was measured in the culture supernatants. Interleukin-1 $\beta$ (IL-1 $\beta$ ), interleukin-6 (IL-6), interleukin-8 (IL-8), interleukin-10 (IL-10), and tumor necrosis factor alpha (TNF- $\alpha$ ) protein levels were measured by a CBA-based assay and reported as pg/ml. Monocytes from healthy subjects (open circle, $n=17$ ) and T2D patients with HbA1c $>9$ (closed circle, $n=12$ ) were stimulated with (a) Pam3Cys or (b) LPS or infected with (c) $M$. tuberculosis. Mann-Whitney $U$ test, healthy subjects vs. T2D patients with HbA1c $>9$. Monocytes from T2D patients and healthy subjects were infected with M. tuberculosis (MOI of 10) for $1 \mathrm{~h}$; after the indicated time, cells were lysed with $1 \%$ SDS, and the mycobacteria were serially diluted and quantified in 7H10 agar media after 21 incubation days, which was reported as CFU. (d) M. tuberculosis growth in healthy subjects (open circle, $n=20$ ) and T2D patients with HbA1c $>9$ (closed circle, $n=19$ ). The median is represented by a horizontal line.

increased susceptibility to $M$. tuberculosis infection, we evaluated the capability of monocytes from T2D patients to control M. tuberculosis intracellular growth. Monocytes were cultured in RPMI medium for 3 days, and the CFU of $M$. tuberculosis were counted on 7H10 agar plates. As shown in Figure 2(d), the intracellular growth of $M$. tuberculosis in monocytes from T2D patients was similar to that in monocytes from healthy subjects (median, $3.8 \times$ $10^{3}-\mathrm{D} 0,3.0 \times 10^{4}-\mathrm{D} 1$, and $2.3 \times 10^{4}-\mathrm{D} 3$ for healthy subjects and $3.9 \times 10^{3}-\mathrm{D} 0,2.5 \times 10^{4}-\mathrm{D} 1$, and $2.1 \times 10^{4}-\mathrm{D} 3$ for T2D patients).

3.5. High Glucose Concentrations Upregulate TLR-2 and TLR-4 Expression in Monocytes from Healthy Subjects.
Because we observed that the TLR expression in T2D patients was not affected, for a next hypothesis, we evaluated whether the glucose per se affects the main innate immune mechanisms for $M$. tuberculosis recognition. We analyzed TLR cell surface expression using an in vitro model of HG (30 mM or $540 \mathrm{mg} / \mathrm{dl}$ glucose). For this experiment, monocytes were cultured in HG for $24 \mathrm{~h}$, and TLR-2 and TLR-4 expression was subsequently determined by flow cytometry. As shown in Figures 3(a)-3(d), both TLR-2 (median, RPMI medium 15.35 and HG 51.28) and TLR-4 (median, RPMI medium 14.27 and HG 30.03) cell surface expression was upregulated significantly in monocytes cultured in HG compared to cells cultured with RPMI medium $(P<0.05)$. 


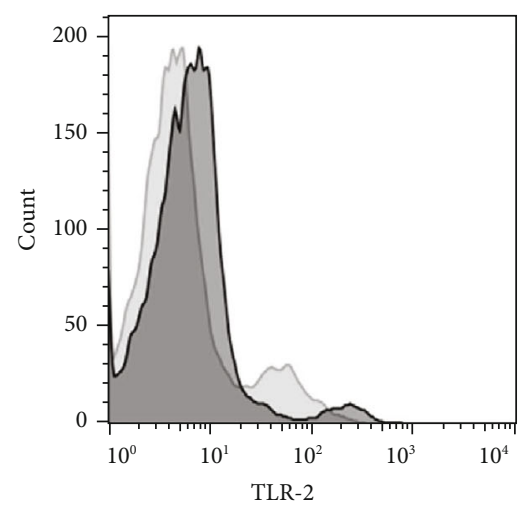

(a)

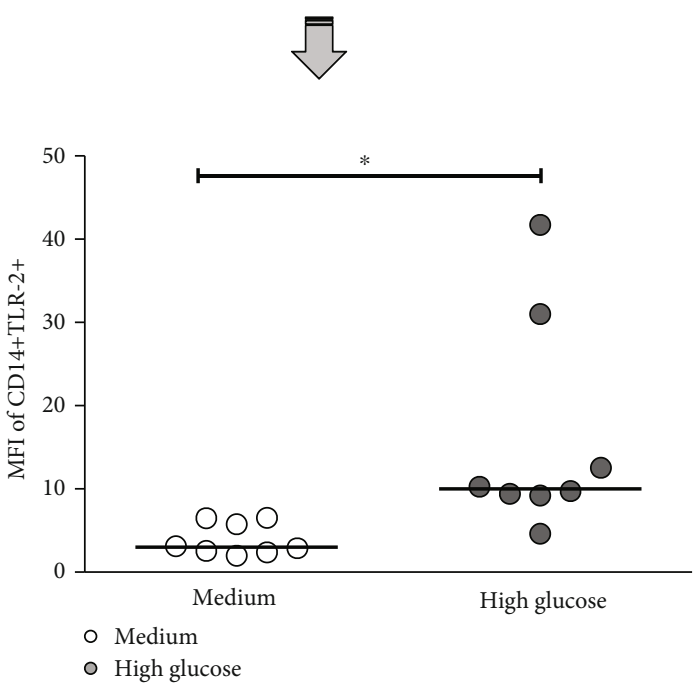

(c)

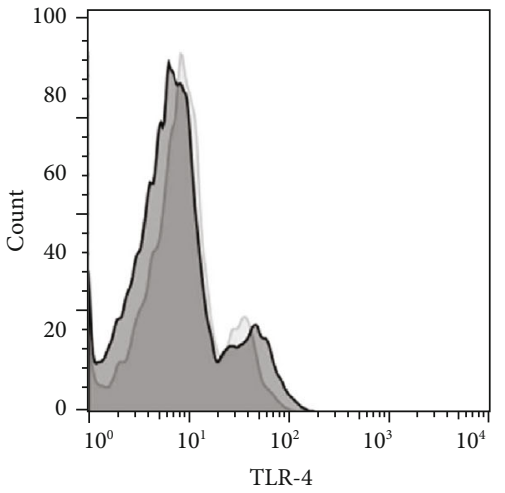

(b)

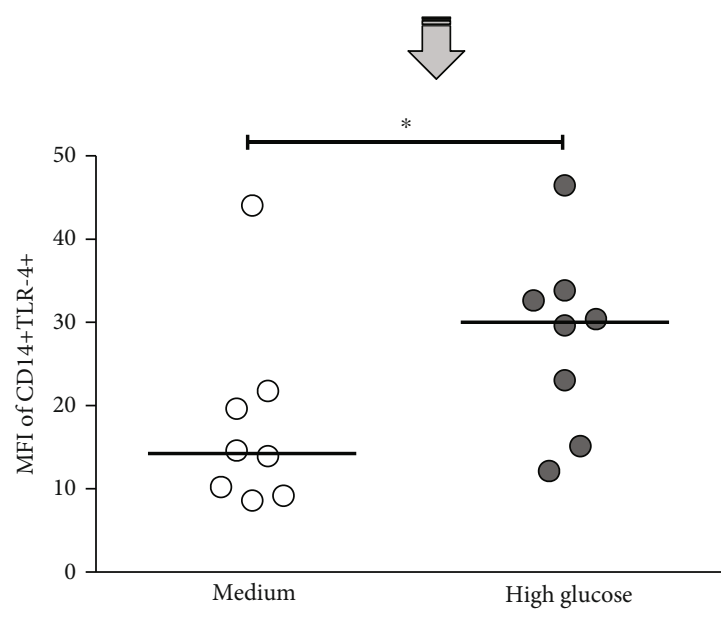

(d)

Figure 3: Effect of high glucose on TLR-2 and TLR-4 surface expression in human monocytes from healthy subjects. Monocytes were cultured in high glucose $(30 \mathrm{mM})$ or RPMI medium for $24 \mathrm{~h}$ and then stained with specific monoclonal antibodies against human CD14, and the TLR and monocyte regions were analyzed. A representative analysis of the effect of HG on (a) TLR-2 and (b) TLR-4 is shown. Expression of (c) TLR-2 and (d) TLR-4 in monocytes cultured in RPMI medium (closed circles) or under HG (30 mM) (gray circles) $(n=8)$. Mann-Whitney $U$ test, ${ }^{*} P<0.05$, medium vs. HG. The median is represented by a horizontal line.

\subsection{Elevated Glucose Concentrations Decrease Cytokine} Production in Response to TLR-2 and TLR-4 Ligands or M. tuberculosis Infection. To identify the effect of HG concentration on TLR-specific activation of monocytes, we evaluated cytokine production in monocytes cultured in HG for $24 \mathrm{~h}$ and after stimulation with Pam3Cys or LPS as TLR-2- or TLR-4-specific ligands, respectively, or $M$. tuberculosis infection for $24 \mathrm{~h}$. HG decreased IL-6 production through TLR-2 activation (median, RPMI medium $57085 \mathrm{pg} / \mathrm{ml}$ and $\mathrm{HG} 6521 \mathrm{pg} / \mathrm{ml}, P<0.05$, Figure 4(a)), while the production of cytokines through TLR-4 activation also decreased; IL-1 $\beta$ (median, RPMI medium $10608 \mathrm{pg} / \mathrm{ml}$ and HG $1418 \mathrm{pg} / \mathrm{ml}$ ), IL-6 (median, RPMI medium $99400 \mathrm{pg} / \mathrm{ml}$ and $\mathrm{HG} 7147 \mathrm{pg} / \mathrm{ml}$ ), and IL-8 (median, RPMI medium $95400 \mathrm{pg} / \mathrm{ml}$ and $\mathrm{HG}$ $53115 \mathrm{pg} / \mathrm{ml})$ levels all were decreased $\quad(P<0.05$, Figure 4(b)). Additionally, HG decreased IL-8 production in response to $M$. tuberculosis infection in $\mathrm{HG}$ compared to RPMI medium (median, RPMI medium $95250 \mathrm{pg} / \mathrm{ml}$ and HG $80346 \mathrm{pg} / \mathrm{ml}, P<0.05$, Figure $4(\mathrm{c}))$.
3.7. High Glucose Reduces Intracellular M. tuberculosis Growth in Monocytes. After phagocytosis, macrophages induce antibacterial mechanisms, including cytokine production, that inhibit the intracellular growth of $M$. tuberculosis in monocytes. We evaluated the intracellular M. tuberculosis growth in monocytes cultured in HG. Figure 4(d) shows an increase in intracellular M. tuberculosis growth in monocytes after 3 days of culture with HG with respect to that in monocytes with RPMI medium $(P<0.05)$ (median, RPMI medium: $1.2 \times 10^{3}-\mathrm{D} 0,1.7 \times 10^{3}-\mathrm{D} 1$, and $3.0 \times 10^{3}-\mathrm{D} 3$ and $\mathrm{HG}: 1.2 \times 10^{3}-\mathrm{D} 0,3.4 \times 10^{3}-\mathrm{D} 1$, and $\left.1.1 \times 10^{3}-\mathrm{D} 3\right)$.

\section{Discussion}

T2D is a metabolic disease with high levels of glucose in the blood and an increased risk of developing active TB. To address the immune mechanisms associated with the $M$. tuberculosis response in T2D patients and since TLR-2 and TLR-4 play critical roles in TB infection, we evaluated the 


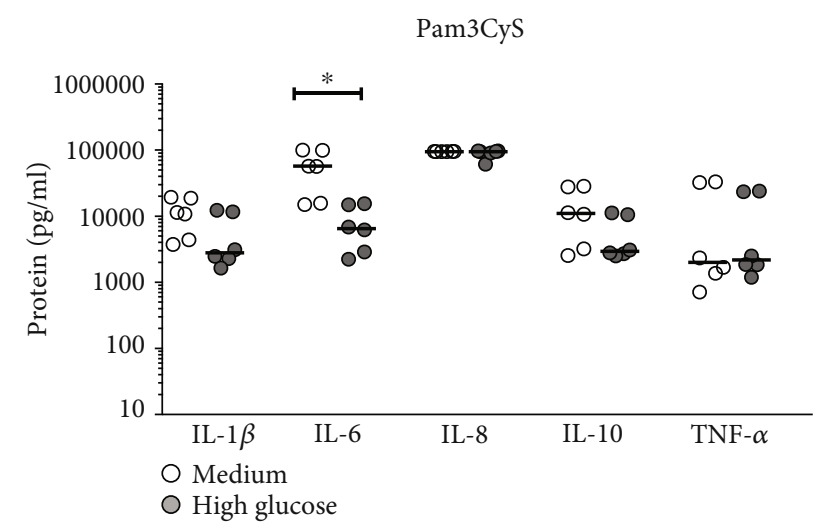

(a)

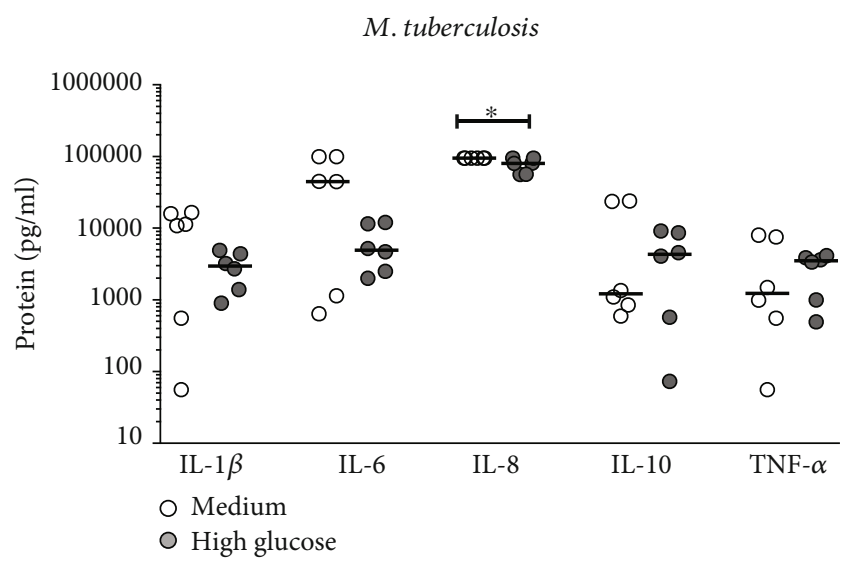

(c)

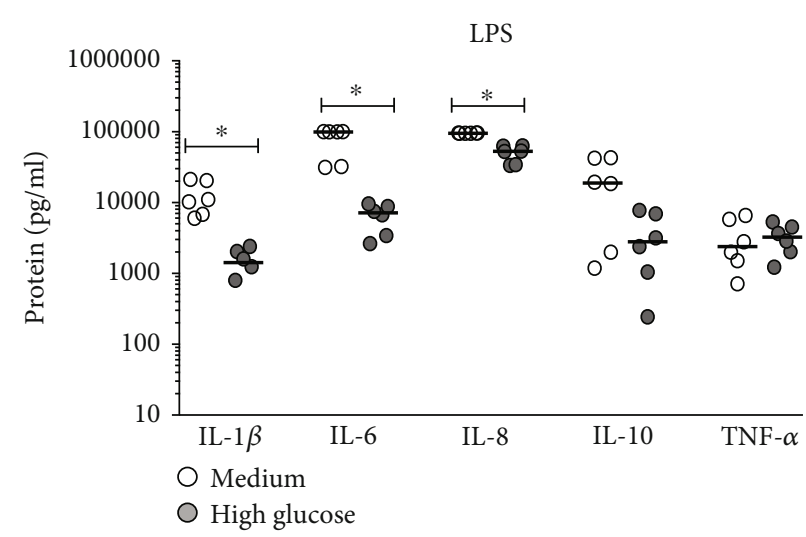

(b)

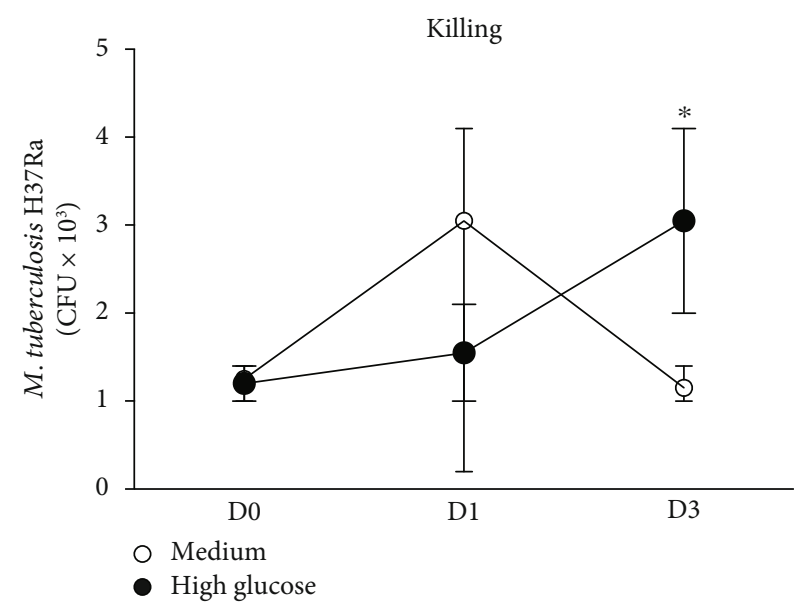

(d)

FIGURE 4: Effect of high glucose on cytokine production after TLR-specific stimulation or M. tuberculosis infection and intracellular growth control of the mycobacteria in human monocytes. Monocytes were infected with M. tuberculosis (MOI of 10) for $1 \mathrm{~h}$. Subsequently, cells were transferred to RPMI medium or HG $(30 \mathrm{mM})$ and cultured for 0,1 , and 3 days. After the indicated time, the supernatants were analyzed for IL- $1 \beta$, IL- 6 , IL- 8 , IL-10, and TNF- $\alpha$ cytokines, as determined by the CBA-based assay. The cells were lysed with $1 \%$ SDS, and the mycobacteria were serially diluted and plated on 7H10 agar media. After 21 days of incubation, CFU were determined. Cytokine production after (a) P3Cys TLR-2 ligand and (b) LPS TLR-4 ligand stimulation or (c) M. tuberculosis infection in culture supernatants in RPMI medium (open circles) and HG (gray circles) $(n=6)$. The growth control of (d) M. tuberculosis in RPMI medium (open circles) and HG (closed circles) is shown $(n=6)$. Mann-Whitney $U$ test, ${ }^{*} P<0.05$, RPMI medium vs. HG. The median is represented by a horizontal line.

TLR expression, proinflammatory cytokine production, and intracellular growth of $M$. tuberculosis in monocytes from T2D patients to assess the intrinsic impairment of monocytes in $M$. tuberculosis recognition and control. We also used an in vitro hyperglycemia model $(30 \mathrm{mM}$ glucose) to assess whether high glucose impaired the control of $M$. tuberculosis. In this population, we did not find a significant difference in TLR-2 and TLR-4 expression, cytokine production, or intracellular growth of M. tuberculosis in T2D patients compared to healthy subjects. However, using the in vitro model of hyperglycemia at $30 \mathrm{mM}$ glucose, we found that cytokine production was diminished after TLR- 2 and TLR- 4 activation and that the intracellular growth of M. tuberculosis in human monocytes was not controlled.

TLRs are an important mechanism for the recognition of M. tuberculosis; TLR-2 and TLR-4 play critical roles in TB infection and are the immediate mechanism for the recognition of pathogens $[15,16]$. Also, TLRs are important regulators of the immune and metabolic systems, including in DM
[17]. However, the role of TLRs in diabetes is controversial; these apparent discrepancies could be explained by the population, experimental design, and type of cell. Dasu et al. report an increased TLR-2 and TLR-4 expression in monocytes from recently diagnosed type 2 diabetic patients [9]. Ahmad et al. report an increased expression of TLR-2 and TLR-4 in PBMCs from obese and overweight individuals with type 2 diabetes [18]. Gupta et al. report no differences in TLR-2, TLR-4, and TLR-6 levels in monocytes from patients with good glycemic control when compared to nondiabetic volunteers [19]. In addition, it was reported that the expression of TLRs did not differ significantly between T2D patients and healthy voluntaries, although their monocytes are less responsive to TLR ligands [20]. Using the whole blood assay to preserve physiological environment and avoid possible artificial stimulation of monocytes, Khondkaryan et al. evaluate the expression of TLR-2 and TLR-4 on the total monocyte surface in culture from the whole blood and report significantly reduced TLR-4 expression in 
unstimulated T2DM monocytes compared to respective cells from healthy donors [21]. In concordance with Khondkaryan et al., we observed that T2D patients express TLR-2 and TLR-4 in monocytes at the same level as healthy subjects; although the healthy population was overweight, a previous report demonstrated that subjects with T2D had significantly elevated mRNA levels of TLR-2 and TLR-4 compared with nondiabetic obese subjects [18]. These contradictory results show that the levels of TLR-2 and TLR-4 are not a hallmark of T2D patients and the levels of TLR expression are rather associated with factors such as glucose levels.

Additionally, in in vitro studies, HG increased the expression of TLR- 2 and TLR- 4 in THP1 cells and primary monocytes from healthy subjects via protein kinase C (PKC) [7]. We also observed the in vitro effect of $\mathrm{HG}$ on the upregulated expression of TLR- 2 and TLR- 4 on the cell surface of primary monocytes from healthy subjects, similar to recently diagnosed type 2 diabetic patients [9], supporting the upregulation of TLRs in the presence of HG concentrations.

After pathogen recognition, TLRs activate signaling cascades via NF- $\kappa$ B to induce proinflammatory responses. The role of hyperglycemia as a proinflammatory factor was demonstrated by in vitro models. In THP-1 monocytes, HG concentrations $(15 \mathrm{mM})$ induce the production of TNF- $\alpha$, interleukin- (IL-) $1 \beta$, chemokines, such as monocyte chemoattractant protein-1 (MCP-1), and interferon gamma inducible protein-10 (IP-10), many of which are NF- $\kappa$ B regulated [22]. Previous reports on models of diabetic rats infected with $M$. tuberculosis showed a reduced production of TNF- $\alpha$, IFN- $\gamma$, and IL-12 by alveolar macrophages [23]. In contrast, in patients with diabetes who had poorly controlled blood glucose levels, TNF- $\alpha$, IFN- $\gamma$, IL- 2 , and IL- $1 \beta$ cytokine levels were increased in response to PPD [24]. However, the response was different when we used the live $M$. tuberculosis H37Ra strain. We observed similar levels of TNF- $\alpha$, IL- $1 \beta$, IL- 6 , and IL- 8 cytokine production, whereas in response to Pam3Cys, a synthetic ligand of TLR-2, only IL-10 production was reduced in T2D patients. A similar response was reported in diabetic mice, with low levels of IL-10 expression in macrophages from diabetic mice [25]. Additionally, HG decreases the release of IL- $1 \beta$, IL- 6 , and IL-8 in LPS-stimulated monocytes cultured in the presence of HG. These results are partially consistent with Beitland et al. who found that IL- $1 \beta$ production was significantly reduced in LPS-stimulated blood samples in the presence of $20 \mathrm{mmol} / \mathrm{l}$ glucose [26]. However, the TLR responses differ in the type and magnitude of the innate immune responses they elicit when interacting with different ligands. We used live $M$. tuberculosis to evaluate the response by monocytes in HG and observed that IL-8 production was significantly reduced. Interleukin-8 (IL-8) displays two major biological activities: chemoattraction and activation of several types of white blood cells. IL-8 plays a central role in the normal immune response to $M$. tuberculosis and has been shown to be absolutely required for granuloma formation. Monocytes and macrophages infected with $M$. tuberculosis are the primary producers of IL- 8 during the course of TB disease, an inability to stimulate the production of IL- 8 corre- lated with a poor prognosis in patients with TB [27], and the reduction in IL-8 production by HG could participate in tuberculosis susceptibility.

It has been well established that recognition and phagocytosis of M. tuberculosis induce the production of antimicrobial peptides, which are important for the control of the intracellular growth of pathogens, including M. tuberculosis [28]. We observed that the growth of $M$. tuberculosis increased in monocytes cultured in HG concentrations. The reduced cytokine production observed in this study suggests inadequate activation through these receptors, which may be a putative mechanism of the reduction in TLR-mediated $M$. tuberculosis control. These findings are consistent with previous studies in animal models of diabetes, in which mice infected with $M$. tuberculosis did not control the infection and died in a short time [29]. Additionally, diabetic rats (with HG levels in their urine) had a large number of granulomas, with a high Mycobacterium load [23]. Our study presents evidence of the role of hyperglycemia in the mechanism associated with $M$. tuberculosis intracellular control.

\section{Conclusion}

TLR- 2 and TLR- 4 expression, cytokine production, and the intracellular growth of $M$. tuberculosis are not associated with an intrinsic factor in monocytes from T2D patients; however, the presence of high glucose concentrations decreased cytokine production and increased the intracellular growth of $M$. tuberculosis in monocytes, suggesting that hyperglycemia at high levels may play a key role in altering the immune responses to $M$. tuberculosis in diabetic patients.

\section{Data Availability}

The research data used during this study are included in the paper. The clinical and demographic data for each patient or healthy subject analyzed in this study are available from the corresponding author upon request.

\section{Ethical Approval}

The protocol was approved by the Ethics Committee in Research of the Instituto Nacional de Enfermedades Respiratorias Ismael Cosío Villegas with reference number B20-12.

\section{Consent}

All participants provided written informed consent.

\section{Conflicts of Interest}

The authors declare that there is no conflict of interest regarding the publication of this article.

\section{Authors' Contributions}

MTH performed the experiments and data analysis, GFM selected the patients, and MT participated in the study design. YG participated in data mining and interpretation 
and wrote the manuscript. All authors reviewed and approved the final manuscript.

\section{Acknowledgments}

We thank all subjects who participated in this study. This study was supported by the Mexican Secretariat of Health and the Mexican Council of Science and Technology (CONACYT) grant CB 2008-101948.

\section{Supplementary Materials}

Correlation of cytokine production and $\mathrm{BMI}$ in T2D patients and healthy subjects. The whole blood from T2D (HbA1c $>9 \%)$ and healthy subjects was stimulated with Pam3Cys (TLR-2 ligand) or LPS (TLR-4 ligand) or infected with $M$. tuberculosis (MOI of 10) and incubated for $4 \mathrm{~h}$. Supernatants were collected, and IL- $1 \beta$, IL-6, IL-8, IL-10, and TNF- $\alpha$ levels were measured by a cytometric bead array, and the cytokine concentration was reported as $\mathrm{pg} / \mathrm{ml}$. Correlation was done between cytokine concentration and body mass index (BMI) using the Spearman test. The dot graphics represent (a) T2D patients (close circle, $n=13$ ) and (b) healthy subjects (open circle). (Supplementary Materials)

\section{References}

[1] World Health Organization, Global tuberculosis report 2018, World Health Organization, Geneva, Switzerland, 2018.

[2] C. Y. Jeon and M. B. Murray, "Diabetes mellitus increases the risk of active tuberculosis: a systematic review of 13 observational studies," PLoS Medicine, vol. 5, no. 7, p. e152, 2008.

[3] M. A. Baker, A. D. Harries, C. Y. Jeon et al., "The impact of diabetes on tuberculosis treatment outcomes: a systematic review," BMC Medicine, vol. 9, no. 1, 2011.

[4] M. E. Jiménez-Corona, L. P. Cruz-Hervert, L. García-García et al., "Association of diabetes and tuberculosis: impact on treatment and post-treatment outcomes," Thorax, vol. 68, no. 3, pp. 214-220, 2013.

[5] K. E. Dooley and R. E. Chaisson, "Tuberculosis and diabetes mellitus: convergence of two epidemics," The Lancet Infectious Diseases, vol. 9, no. 12, pp. 737-746, 2009.

[6] M. Turina, D. E. Fry, and H. C. Polk Jr., "Acute hyperglycemia and the innate immune system: clinical, cellular, and molecular aspects," Critical Care Medicine, vol. 33, no. 7, pp. 16241633, 2005.

[7] M. R. Dasu, S. Devaraj, L. Zhao, D. H. Hwang, and I. Jialal, "High glucose induces Toll-like receptor expression in human monocytes mechanism of activation," Diabetes, vol. 57, no. 11, pp. 3090-3098, 2008.

[8] E. Mortaz, I. M. Adcock, P. Tabarsi et al., "Interaction of pattern recognition receptors with Mycobacterium tuberculosis," Journal of Clinical Immunology, vol. 35, no. 1, pp. 1-10, 2015.

[9] M. R. Dasu, S. Devaraj, S. Park, and I. Jialal, "Increased Tolllike receptor (TLR) activation and TLR ligands in recently diagnosed type 2 diabetic subjects," Diabetes Care, vol. 33, no. 4, pp. 861-868, 2010.

[10] S. M. Reyna, S. Ghosh, P. Tantiwong et al., "Elevated Toll-like receptor 4 expression and signaling in muscle from insulinresistant subjects," Diabetes, vol. 57, no. 10, pp. 2595-2602, 2008.
[11] Y. Gonzalez, M. T. Herrera, G. Soldevila et al., "High glucose concentrations induce TNF- $\alpha$ production through the downregulation of CD33 in primary human monocytes," $B M C$ Immunology, vol. 13, no. 1, p. 19, 2012.

[12] S. Mirza, M. Hossain, C. Mathews et al., "Type 2-diabetes is associated with elevated levels of TNF-alpha, IL-6 and adiponectin and low levels of leptin in a population of Mexican Americans: a cross-sectional study," Cytokine, vol. 57, no. 1, pp. 136-142, 2012.

[13] J. J. Kim and D. D. Sears, "TLR4 and insulin resistance," Gastroenterology Research and Practice, vol. 2010, Article ID 212563, 11 pages, 2010.

[14] American Diabetes Association, "2. Classification and Diagnosis of Diabetes," Diabetes Care, vol. 38, Supplement_1, pp. S8S16, 2015.

[15] S. E. Doyle, R. M. O'Connell, G. A. Miranda et al., "Toll-like receptors induce a phagocytic gene program through P38," The Journal of Experimental Medicine, vol. 199, no. 1, pp. 81-90, 2004.

[16] M. A. Sanjuan, C. P. Dillon, S. W. G. Tait et al., "Toll-like receptor signalling in macrophages links the autophagy pathway to phagocytosis," Nature, vol. 450, no. 7173, pp. $1253-$ 1257, 2007.

[17] W. Kanczkowski, C. G. Ziegler, K. Zacharowski, and S. R. Bornstein, "Toll-like receptors in endocrine disease and diabetes," Neuroimmunomodulation, vol. 15, no. 1, pp. 54-60, 2008.

[18] R. Ahmad, A. al-Mass, V. Atizado et al., "Elevated expression of the Toll like receptors 2 and 4 in obese individuals: its significance for obesity-induced inflammation," Journal of Inflammation, vol. 9, no. 1, p. 48, 2012.

[19] S. Gupta, A. Maratha, J. Siednienko et al., "Analysis of inflammatory cytokine and TLR expression levels in type 2 diabetes with complications," Scientific Reports, vol. 7, no. 1, 2017.

[20] T. Komura, Y. Sakai, M. Honda, T. Takamura, K. Matsushima, and S. Kaneko, "CD14+ monocytes are vulnerable and functionally impaired under endoplasmic reticulum stress in patients with type 2 diabetes," Diabetes, vol. 59, no. 3, pp. 634-643, 2010.

[21] L. Khondkaryan, S. Margaryan, D. Poghosyan, and G. Manukyan, "Impaired inflammatory response to LPS in type 2 diabetes mellitus," International Journal of Inflammation, vol. 2018, 6 pages, 2018.

[22] N. Shanmugam, M. A. Reddy, M. Guha, and R. Natarajan, "High glucose-induced expression of proinflammatory cytokine and chemokine genes in monocytic cells," Diabetes, vol. 52, no. 5, pp. 1256-1264, 2003.

[23] I. Sugawara, H. Yamada, and S. Mizuno, "Pulmonary tuberculosis in spontaneously diabetic Goto Kakizaki rats," The Tohoku Journal of Experimental Medicine, vol. 204, no. 2, pp. 135-145, 2004.

[24] B. I. Restrepo, S. P. Fisher-Hoch, P. A. Pino et al., "Tuberculosis in poorly controlled type 2 diabetes: altered cytokine expression in peripheral white blood cells," Clinical Infectious Diseases, vol. 47, no. 5, pp. 634-641, 2008.

[25] M. K. Mohammad, M. Morran, B. Slotterbeck et al., "Dysregulated Toll-like receptor expression and signaling in bone marrow-derived macrophages at the onset of diabetes in the non-obese diabetic mouse," International Immunology, vol. 18, no. 7, pp. 1101-1113, 2006. 
[26] S. Beitland, H. Opdahl, T. Aspelin, L. Saetre, and T. Lyberg, "Blood leucocyte cytokine production after LPS stimulation at different concentrations of glucose and/or insulin," Acta Anaesthesiologica Scandinavica, vol. 53, no. 2, pp. 183-189, 2009.

[27] A. Krupa, M. Fol, B. R. Dziadek et al., "Binding of CXCL8/IL-8 to Mycobacterium tuberculosis modulates the innate immune response," Mediators of Inflammation, vol. 2015, 11 pages, 2015.

[28] J. Basu, D.-M. Shin, and E.-K. Jo, "Mycobacterial signaling through Toll-like receptors," Frontiers in Cellular and Infection Microbiology, vol. 2, 2012.

[29] O. Saiki, S. Negoro, I. Tsuyuguchi, and Y. Yamamura, "Depressed immunological defense mechanisms in mice with experimentally induced diabetes," Infection and immunity, vol. 28, no. 1, pp. 127-131, 1980. 


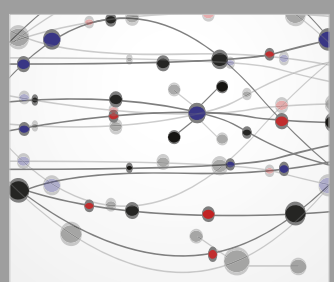

The Scientific World Journal
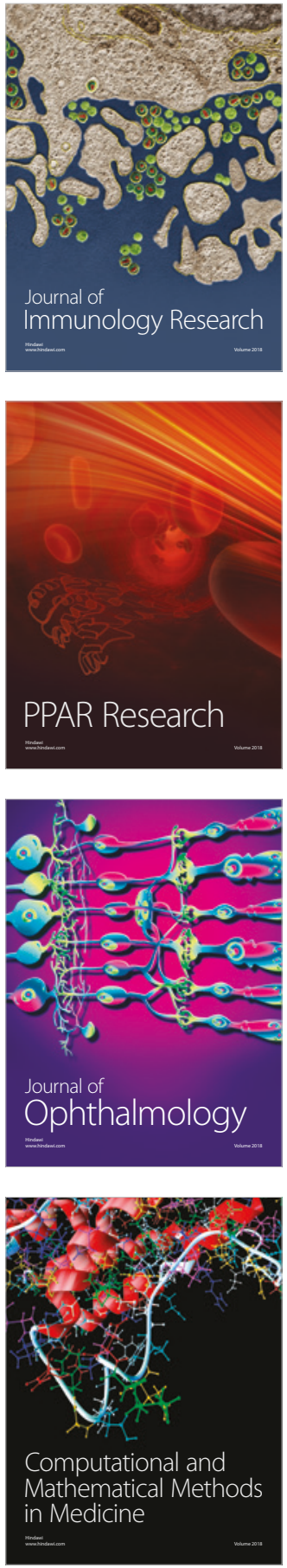

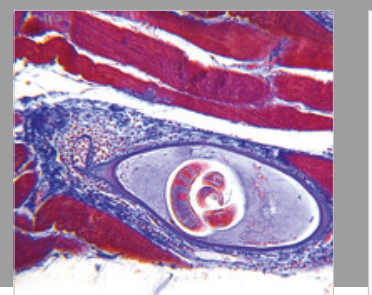

Gastroenterology Research and Practice

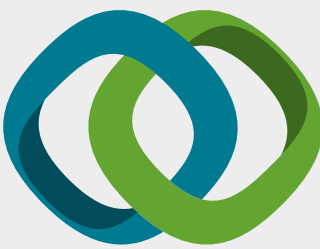

\section{Hindawi}

Submit your manuscripts at

www.hindawi.com
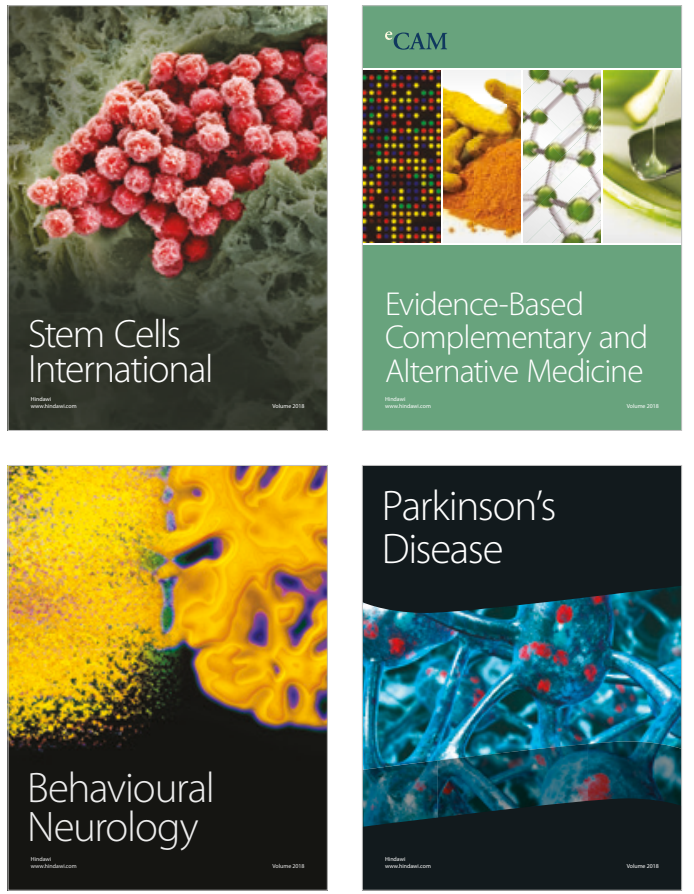

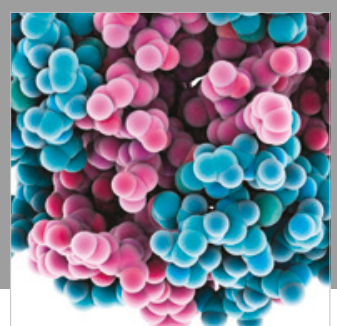

ournal of

Diabetes Research

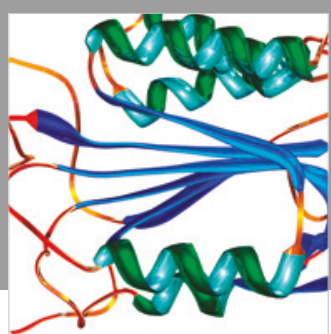

Disease Markers
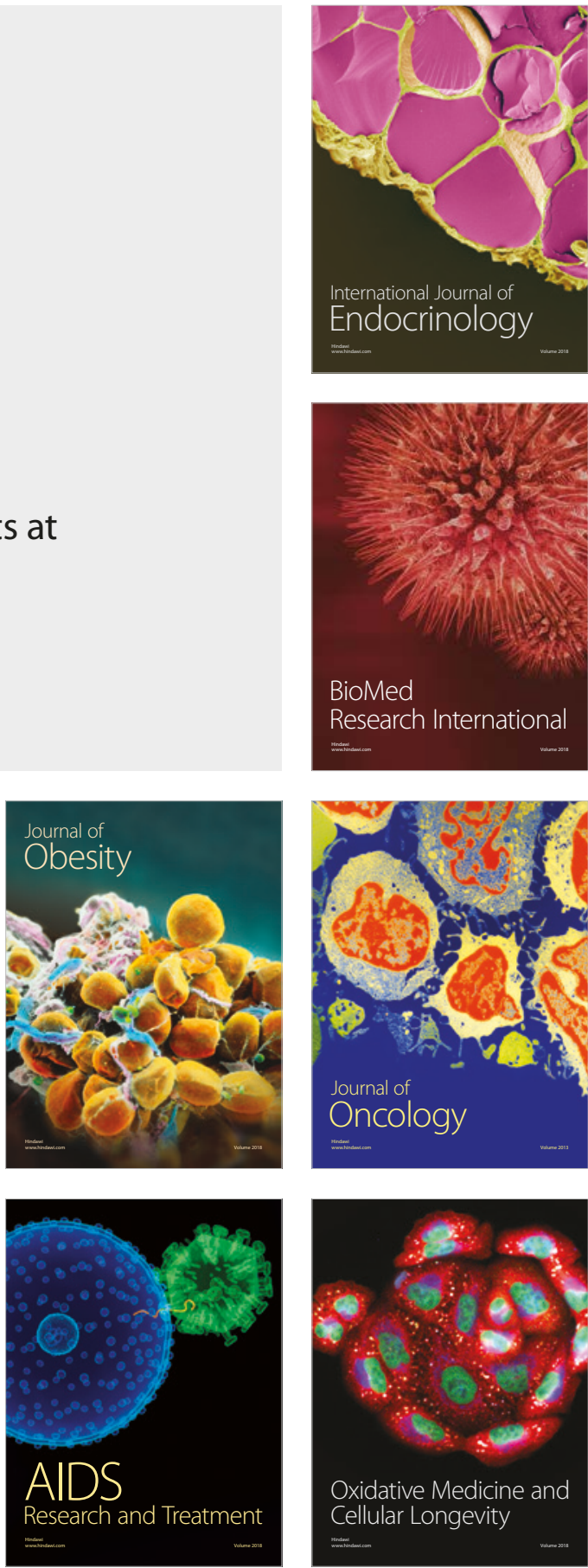